\title{
Estudio de conformidad con la formación recibida por los residentes del Hospital Universitario Príncipe de Asturias de 2007 a 2009
}

\author{
José A. Gómez-Carrasco, Paula Pais-Roldán
}

Introducción. El Real Decreto 183/2008 destaca la importancia de la evaluación formativa en el sistema de residencia para la formación especializada. En evaluación formativa lo importante es el feedback producido sobre el que aprende y el que enseña, contribuyendo así a la mejora del proceso enseñanza-aprendizaje. Una parte esencial de la evaluación formativa es la pregunta directa a los sujetos a quienes se dirige la formación.

Sujetos y métodos. Desde junio de 2007, los residentes del hospital cumplimentan un cuestionario de 37 preguntas cerradas y una abierta, en la que se les solicitaba su opinión sobre aspectos de la formación recibida en el año precedente. Analizamos los cuestionarios de tres años (266 encuestas analizadas).

Resultados. Las guardias y las urgencias en general se perciben como un problema y como un lugar donde hay una importante área de mejora. Otras importantes áreas de mejora son la capacitación del residente para optimizar el acceso a fuentes de información y una mayor participación e implicación en investigación.

Conclusiones. Estudios como el descrito deben servir para implantar acciones eficientes orientadas a mejorar las deficiencias observadas en la formación de residentes. Ha de ser punto de referencia para conocer la evolución del proceso formativo en nuestro centro. Si no se realizan este tipo de estudios es difícil conocer los progresos o deficiencias que, desde el punto de vista de quien aprende, tienen lugar en un determinado ámbito de la formación de especialistas en ciencias de la salud.

Palabras clave. Evaluación formativa. Formación de residentes. Formación especializada.

A study of conformity with the training received by resident physicians at the Hospital Universitario Príncipe de Asturias between 2007 and 2009

Introduction. The Royal Decree 183/2008 highlights the relevance of the formative assessment in the residence system for specialist training. In formative assessment, the most important is the feedback between learner and teacher, contributing so to the continuous improvement in teach-learning process. An essential part of the formative assessment is the direct ask posed to whom the formation is directed.

Subjects and methods. Since June 2007, the residents filled a survey which includes 37 closed questions, and an one open question. In this questionnaire we expect their opinion about aspects in the education received in the past year. We analyzed the questionnaires of three years (total 266 surveys were analyzed).

Results. Medical guards and emergency area were perceived as an important problem, as a place for improvement. Other relevant areas for improvement were the training for residents' ability for effective bibliographic researches, and greater involvement in research activities.

Conclusions. Studies like these should be used to implement efficient actions aimed to detect problems and to improve deficiencies in residents' training. It has to be a reference point for following the learning evolution process in our center. Failure to make such studies it will be difficult to know the progress or deficiencies, from the learner standpoint, that take place in a particular area of training of specialists in health sciences.

Key words. Formative assessment. Specialist training. Teaching residents.
Jefe de Estudios del Hospital Universitario Príncipe de Asturias y Departamento de Especialidades Médicas de la Universidad de Alcalá (J.A. Gómez-Carrasco). Estudiante de 6. curso de Licenciatura de Medicina de la Universidad de Alcalá (P. Pais-Roldán). Alcalá de Henares, Madrid, España.

Correspondencia:

Prof. José Ángel Gómez Carrasco. Comisión Local de Docencia. Hospital Universitario Príncipe de Asturias. Carretera de Meco, $s / n$. E-28805 Alcalá de Henares (Madrid).

E-mail:

jgomez.hupa@salud.madrid.org

Conflicto de intereses:

No declarado.

Competing interests: None declared.

(c) 2012 Educación Médica 


\section{Introducción}

La formación posgraduada es un fenómeno complejo en el que están implicadas muchas personas y estructuras. Esta formación asume con la sociedad una gran responsabilidad porque finalizará con el retorno al mundo del trabajo de profesionales que atenderán a la salud de los ciudadanos durante algunas décadas. El proceso de formación necesita ser evaluado. Convencidos del papel central de la evaluación, afirmamos que sin una medición o evaluación educativa rigurosa no puede haber una educación eficiente. 'Medir con precisión para enseñar con rigor' es el lema actual en evaluación [1]. Desde una evaluación centrada en el rendimiento, las tendencias actuales reducen la importancia de los objetivos y se desplazan hacia las necesidades educativas. Así emergen los conceptos de evaluación sumativa y evaluación formativa (EF). Ésta pone el acento no sólo sobre metas y objetivos, sino también sobre contenidos, procesos, procedimientos y actitudes, tanto de quien enseña como de quien aprende. En EF, los buenos docentes utilizan la calificación para ayudar a los estudiantes a aprender y no sólo para clasificar y jerarquizar; la tarea del evaluador es obtener y comunicar una amplia información que permita aflorar dificultades del aprendizaje. La EF trata de responder a la cuestión: ¿qué tipo de desarrollo intelectual y personal quiero que disfruten los que aprenden y qué evidencias podré obtener sobre la naturaleza y el progreso de ese desarrollo? [2]. El Real Decreto 183/2008 que regula la formación de residentes, en su artículo 17 , destaca la adecuación de la EF y el importante papel que ésta adquiere en el sistema de residencia para la formación especializada [3]. Esta opinión es compartida hoy por la totalidad de líderes de opinión en formación médica $[4,5]$.

En EF, lo verdaderamente importante es el feedback producido sobre el aprendiz, asumiéndose que contribuirá a la mejoría del proceso de enseñanzaaprendizaje. No obstante, aun hoy, no queda claro si a través de la multitud de métodos propuestos para llevar a cabo EF en el ámbito de las profesiones sanitarias, bien sea en periodo de formación o posteriormente, el feedback producido mejora el rendimiento $[6,7]$.

Los profesores auténticamente buenos intentarán averiguar tantas cosas de sus estudiantes como les sea posible, 'no tanto para enjuiciarlos', sino 'porque así podré ayudarlos a aprender' [2]. La EF será inevitablemente personalizada, de acuerdo con los intereses y aptitudes de cada alumno, y se efectuará mediante herramientas diversas, como esca- las de observación, informes de observadores externos, análisis de tareas, estudios de casos, etc., pero también mediante entrevistas y cuestionarios. En EF se da voz a todos los participantes en el proceso formativo, tanto docentes como discentes, obteniendo así información útil sobre la totalidad del proceso, siendo a su vez punto de partida para elaborar estrategias de mejora continua de la calidad. Por ello, una parte esencial, aunque no única, de la evaluación del proceso es la pregunta directa a los sujetos a los que va destinada la formación.

Por último, el grado de evaluación y cumplimiento de objetivos deberá publicitarse de forma general para que los aspirantes a la formación especializada tengan conocimiento del nivel de logros alcanzados por las diferentes unidades docentes. Una de las obligaciones normativas de las comisiones de docencia de los hospitales y centros de formación de residentes es 'promover la evaluación anual, por parte de los residentes, de la estructura y la actividad docente' [8].

Hasta la actualidad, en nuestro hospital no ha tenido lugar de forma sistemática la evaluación de las unidades docentes por parte de los propios residentes que se forman en ellas. Realizar una primera evaluación de la formación en nuestro hospital universitario de la Universidad de Alcalá es el objetivo principal de este estudio.

\section{Ámbito del estudio}

El estudio se llevó a cabo en el Hospital Universitario Príncipe de Asturias (HUPA), un hospital general con clara vocación asistencial y docente, que presta asistencia sanitaria especializada a una población de 376.274 habitantes; sus municipios de referencia principales son Alcalá de Henares y Torrejón de Ardoz. Es el hospital del Ârea Sanitaria III de la Comunidad Autónoma de Madrid y hospital universitario de la Facultad de Medicina de la Universidad de Alcalá. Desde 1990 cuenta con la acreditación para la formación de residentes. Desde entonces ha formado a más de 600 profesionales. En la actualidad tiene acreditadas las unidades docentes que se enumeran en la tabla II, una Unidad Docente Multiprofesional de Salud Mental, y contribuye a la formación de residentes de medicina familiar y comunitaria (MFyC) (18 residentes/año) colaborando con la unidad docente de la especialidad. La acreditación en Dermatología y Reumatología es reciente, por lo que hasta la actualidad no hemos tenido ningún residente en dichas especialidades que haya cursado un año completo de formación. 


\section{Sujetos y métodos}

Desde junio de 2007, los residentes del HUPA cumplimentan anualmente un cuestionario que contiene 37 preguntas cerradas y una abierta, en el que se les solicita opinión sobre determinados aspectos de la formación recibida a lo largo del año precedente. La Comisión Local de Docencia (CLD) del HUPA dispone de las encuestas cumplimentadas por los residentes, referidas a los años 2007, 2008 y 2009.

El objetivo principal de este estudio es extractar la información que estos cuestionarios aportan sobre el proceso formativo en el periodo de residencia. Esta información se procesó según el año de residencia y especialidad. La información de este estudio debe ser útil para reflexionar sobre las carencias percibidas, así como punto de partida para implantar mejoras en dicho proceso.

Las diferentes variables se agrupan en los siguientes campos:

- Relativo a la unidad docente a la que pertenece: solicitamos una valoración global de la unidad e información sobre sesiones clínicas, elaboración de comunicaciones a congresos, dedicación a la actividad asistencial, el acceso a fuentes de información y la supervisión de su actividad asistencial.

- Relativo a unidades, servicios o secciones por las que ha rotado en este año/curso evaluado.

- Relativo a la labor tutorial.

- Relativo a la acogida en el hospital y unidad docente.

- Comentarios libres en relación a la docencia recibida, interesando particularmente señalar aspectos y áreas de mejora.

Las variables del estudio se han introducido en un fichero Access, el cual se ha exportado al programa SPSS v. 15.0, realizándose el análisis estadístico pertinente: descriptivo para variables cuantitativas y cualitativas, estadística analítica para comparar medias de variables cuantitativas, principalmente mediante la prueba de ANOVA para varias muestras., y test de chi al cuadrado para análisis de las diferencias entre proporciones. El apartado de comentarios libres ha sido objeto de un análisis conceptual y cualitativo de las principales ideas expuestas por los residentes, y se han agrupado en los clásicos campos de asistencia, docencia e investigación.

Del análisis estadístico se han excluido las cuestiones relativas a las rotaciones por unidades, servicios o secciones por las que los residentes rotan, dado que son muchas, con denominaciones muy dispares y desarrolladas bajo la tutela de muy diferentes facultativos, todo lo cual impide un análisis estadís-

\begin{tabular}{lcc} 
Tabla I. Año de residencia $(n=266)$. & \\
\hline MIR-I & $n$ & $\%$ \\
\hline MIR-II & 81 & 30,5 \\
\hline MIR-III & 67 & 25,2 \\
\hline MIR-IV & 68 & 25,6 \\
\hline MIR-V & 42 & 15,8 \\
\hline
\end{tabular}

Tabla II. Especialidad MIR $(n=266)$.

\begin{tabular}{|c|c|c|}
\hline & $n$ & $\%$ \\
\hline Análisis clínicos & 6 & 2,3 \\
\hline Anestesiología y reanimación & 10 & 3,8 \\
\hline Aparato digestivo & 7 & 2,6 \\
\hline Cirugía general y del aparato digestivo & 2 & 0,8 \\
\hline Cirugía ortopédica y traumatología & 10 & 3,8 \\
\hline Endocrinología y nutrición & 8 & 3,0 \\
\hline Farmacia hospitalaria & 15 & 5,6 \\
\hline Hematología y hemoterapia & 1 & 0,4 \\
\hline Medicina familiar y comunitaria & 27 & 10,2 \\
\hline Medicina intensiva & 6 & 2,3 \\
\hline Medicina interna & 32 & 12,0 \\
\hline Nefrología & 7 & 2,6 \\
\hline Neurología & 9 & 3,4 \\
\hline Obstetricia y ginecología & 22 & 8,3 \\
\hline Oftalmología & 21 & 7,9 \\
\hline Otorrinolaringología & 12 & 4,5 \\
\hline Pediatría y áreas específicas & 21 & 7,9 \\
\hline Psicología & 15 & 5,6 \\
\hline Psiquiatría & 15 & 5,6 \\
\hline Radiodiagnóstico & 15 & 5,6 \\
\hline Urología & 5 & 1,9 \\
\hline
\end{tabular}


Figura 1. Puntuación de la unidad docente a la que pertenecen, según el nivel de residencia.

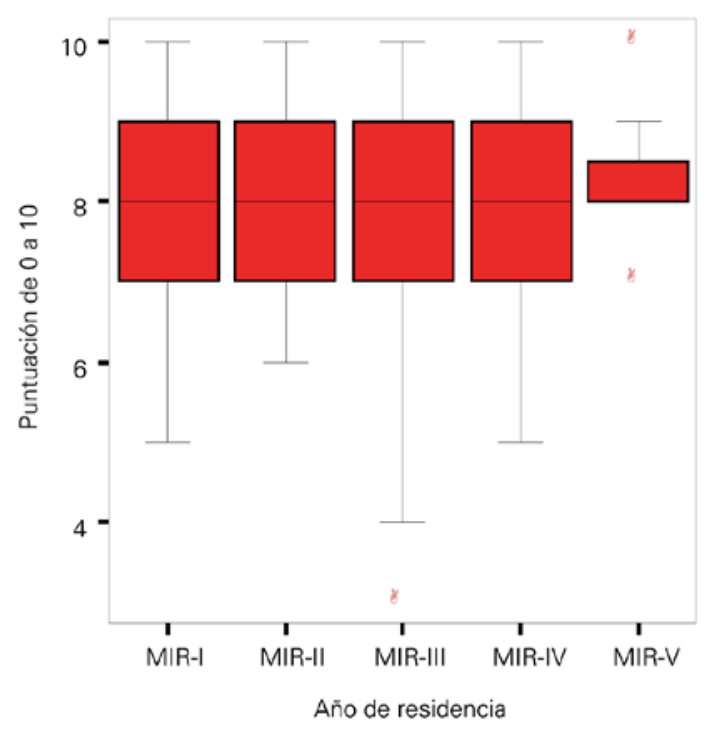

tico clásico. No obstante, los datos se han incluido en el fichero SPSS para intentar realizar análisis parciales por especialidad y rotación, si así se solicitase.

El estudio estadístico se llevó a cabo a lo largo del primer trimestre del 2010 y se han publicitado sus resultados entre tutores y directores de unidades docentes en el segundo trimestre.

\section{Limitaciones del estudio}

Los cuestionarios son respondidos por los residentes al finalizar el año; por tanto, puede haber sesgos de recuerdo. Tampoco se ha supervisado la veracidad de algunos de los datos (p. ej., número de comunicaciones a congresos).

Debe tenerse presente que algunos residentes no han respondido a todas las preguntas, y a pesar de intentar mantener el anonimato de las respuestas, es lógico deducir que, en muchos casos, este anonimato no puede garantizarse totalmente (p. ej., en especialidades con un residente por año) y ello puede desvirtuar algunos de los datos aportados.

\section{Resultados}

\section{Muestra}

Se compone de 266 encuestas de satisfacción, cum- plimentadas por los médicos residentes durante tres años consecutivos (2006-2007, 2007-2008 y 20082009), con 37, 90 y 139 encuestas, respectivamente. En el último curso 2008-2009, la CLD exigió la cumplimentación de la encuesta en el momento de realizar la evaluación anual. En las tablas I y II se aporta el número y las proporciones de las encuestas de residentes por nivel de residencia y especialidades, respectivamente.

\section{Relativo a la unidad docente a la que pertenece}

A la pregunta 'En una escala de 0 a 10, ¿qué puntuación global otorga a la docencia que recibe en la unidad docente objeto de esta evaluación?', el total de la muestra puntuó como sigue: mínima puntuación 3 , máximo 10 , media 7,94, desviación estándar 1,20.

Esta puntuación por años de residencia fue, para los residentes de primer año (MIR-I), 7,96 $\pm 1,23$ (rango: 5-10); para los de segundo año (MIR-II), 8,03 \pm 0,95 (rango: 6-10); para los de tercer año (MIR-III), $7,82 \pm 1,34$ (rango: $3-10$ ); para los de cuarto año, $8,25 \pm 1,34$ (rango: 7-10), y para los de quinto año $(n=8)$, de 8,25 $\pm 0,88$ (rango: 7-10) (Fig. 1). La mediana se situó prácticamente en 8 puntos a lo largo de todo el periodo de formación. La puntuación por especialidades osciló entre una media mínima de 6,47 $\pm 1,68$ de una de las unidades, hasta el máximo de 9,00 \pm 0,47 en otra; en el resto, la puntuación media se situó entre 7,00 y 8,80 (Fig. 2).

Esta puntuación global de las unidades docentes (de 0 a 10), a lo largo de los tres años evaluados, se ha mantenido prácticamente constante en los límites próximos a los 8 puntos: mayo de 2007, 7,92 \pm 0,89; mayo de 2008, 8,03 $\pm 1,28$, y mayo de 2009 , $7,88 \pm 1,23$, sin diferencias estadísticamente significativas ( $p=0,643$, ANOVA).

A la pregunta cerrada 'La valoración global de la unidad docente por la que ha finalizado el curso al que hace referencia esta evaluación, en lo que se refiere a aspectos docentes, puede afirmar que es..,' las respuesta fueron: muy deficiente, $1(0,4 \%)$; deficiente, 6 (2,3\%); adecuada, 46 (17,3\%); buena, 153 (57,5\%); excelente, 54 (20,3\%), y sin respuesta, 6 (2,3\%). Buena o excelente suman un $78 \%$.

\section{Sobre las sesiones clínicas}

¿Con qué frecuencia se organizan sesiones clínicas en su unidad docente?': ocasional o ninguna, $5(1,9 \%)$; mensual, 35 (13,2\%); quincenal, 9 (3,4\%); semanal, $81(30,5 \%)$; más de una vez a la semana, $98(36,8 \%)$, y diaria, $29(10,9 \%)$. 
¿Con qué frecuencia ha podido asistir a las sesiones clínicas?': a ninguna o menos del $25 \%, 11(4,1 \%)$; entre el 25 y 50\%, 31 (11,7\%); a la gran mayoría, 158 $(59,4 \%)$; a todas, $60(22,6 \%)$, y sin respuesta, $6(2,3 \%)$.

¿Ha participado en las sesiones clínicas de forma activa presentando alguna(s)?': no, 24 (9\%); sí, 239 (89,8\%), y sin respuesta, 3 (1,1\%).

¿Cuántas sesiones o casos clínicos ha presentado a lo largo del año/curso evaluado? (cifra exacta o aproximada)': a esta pregunta obtuvimos 262 respuestas y la media fue de $11,2 \pm 41$ (rango: 0-420). Debemos entender que probablemente hay un error de concepto en la idea de 'sesión clínica', que si no se matiza más, puede incluir actividades muy diversas entre residentes de diferentes especialidades. Así, han aportado cifras muy elevadas residentes de cirugía general, radiodiagnóstico, otorrinolaringología, urología y algunos otros. Probablemente la idea de sesión clínica que pretendíamos transmitir no es compatible con cifras de más de 50 por año de formación, y aun así, esta cifra sería elevada. Si excluimos los casos en que se dan cifras superiores a 50 sesiones anuales, casos que probablemente se refieren a actividades diferentes a lo que clásicamente se entiende como una sesión especialmente preparada y expuesta al servicio para el aprendizaje general, el resultado sería de 253 respuestas con una media de 4,68 \pm 5,5 (rango: 0-40).

En la elección, elaboración y presentación de sesiones clínicas, la orientación y apoyo que ha recibido por el tutor (o tutores) ha sido: nula, $6(2,3 \%)$; escasa, 23 (8,6\%); adecuada, 59 (22,2\%); buena, 100 $(37,6 \%)$; excelente, $59(22,2 \%)$, y sin respuesta, 19 $(7,1 \%)$. Buena y excelente suman casi el $60 \%$.

\section{De las contribuciones a congresos}

¿Ha participado en congresos de forma activa presentando alguna comunicación, póster o ponencia?’: respondieron no 91 residentes $(34,2 \%)$, respondieron sí 165 (62\%), y no respondieron 10 (3,8\%).

¿Cuántas comunicaciones, pósters o ponencias ha presentado a lo largo del año/curso evaluado?': se obtuvieron 244 respuestas con una media de 2,24 $\pm 3,49$ (rango: 0-26). Este dato por años de residencia y por año evaluado se ve en las tablas III y IV.

'En la elección, elaboración y presentación de comunicaciones, pósters o ponencias, la orientación y apoyo que ha recibido por el tutor (o tutores)' fue definida como: nula, 8 (3\%); escasa, $11(4,1 \%)$; adecuada, 46 (17,3\%); buena, 68 (25,6\%); excelente, 36 $(13,5 \%)$, y sin respuesta, 97 (36,5\%).

El número de colaboraciones a congresos y cursos por cada residente y por especialidad, en el total

\begin{tabular}{lccc}
\hline \multicolumn{2}{l}{ Tabla III. Colaboraciones a congresos según el año de residencia. } & \\
\hline Año de residencia & $n$ & Media & Desviación estándar \\
\hline MIR-I & 70 & 0,57 & 0,791 \\
\hline MIR-II & 61 & 2,07 & 2,228 \\
\hline MIR-III & 66 & 2,82 & 3,477 \\
\hline MIR-IV & 39 & 4,00 & 4,936 \\
\hline MIR-V & 8 & 4,88 & 8,659 \\
\hline Total & 244 & 2,24 & 3,498
\end{tabular}

Tabla IV. Colaboraciones a congresos según el año evaluado.

\begin{tabular}{lccc}
\hline Fecha de fin de curso & $n$ & Media & Desviación estándar \\
\hline 31.05 .2007 & 37 & 2,73 & 3,687 \\
\hline 31.05 .2008 & 89 & 2,08 & 3,644 \\
\hline 31.05 .2009 & 118 & 2,21 & 3,338 \\
\hline Total & 244 & 2,24 & 3,498 \\
\hline
\end{tabular}

de la muestra, fue muy variable entre las diferentes especialidades (rango: 0-10,4), con una media de $2,24 \pm 3,98$.

\section{De la proporción de jornada laboral dedicada a asistencia 0 a actividades docentes propiamente dichas}

La proporción de jornada reconocida como dedicada a la actividad asistencial fue de $71,94 \pm 16,9 \%$. La proporción de la jornada laboral que dicen dedicar a la docencia propiamente dicha fue de $0,15 \pm 2,4 \%$. Aquí también la variación entre especialidades fue notable (Fig. 3).

\section{De las guardias}

Interrogados sobre el promedio de guardias que realizan mensualmente, la media se situó en 5,08 \pm 1,7 , sin demasiada variabilidad entre especialidades, excepto en aparato digestivo y nefrología porque parte de la atención continuada en estas especialidades se desarrolla en horario vespertino, para endoscopias y hemodiálisis, respectivamente. A la pre- 
Figura 2. Puntuación por unidades docentes.

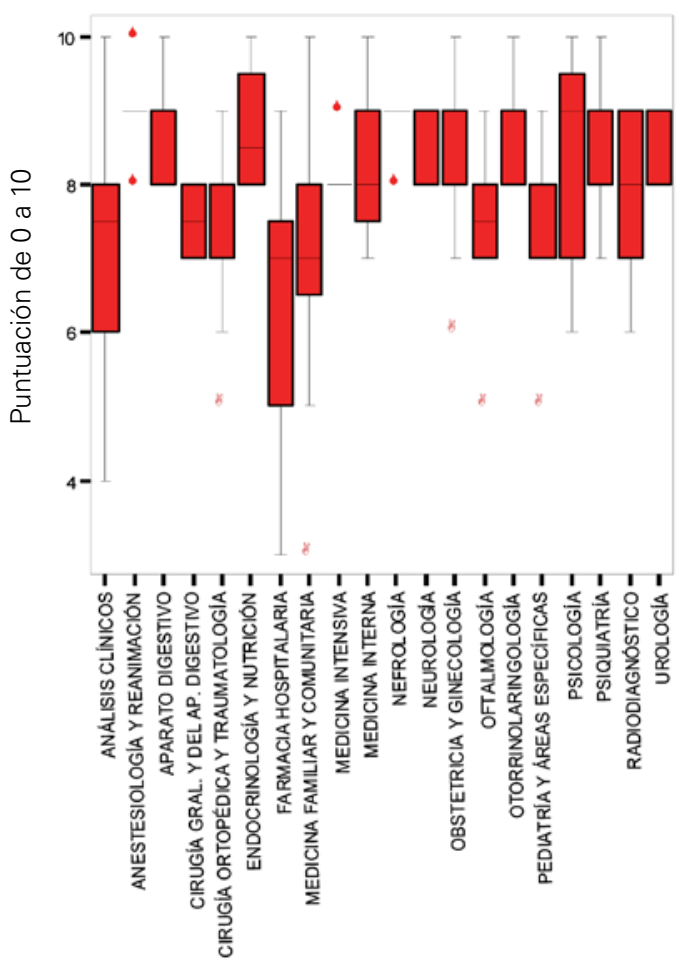

Especialidad MIR
Figura 3. Proporción de la jornada laboral que dicen dedicar a la asistencia propiamente dicha, según la especialidad.

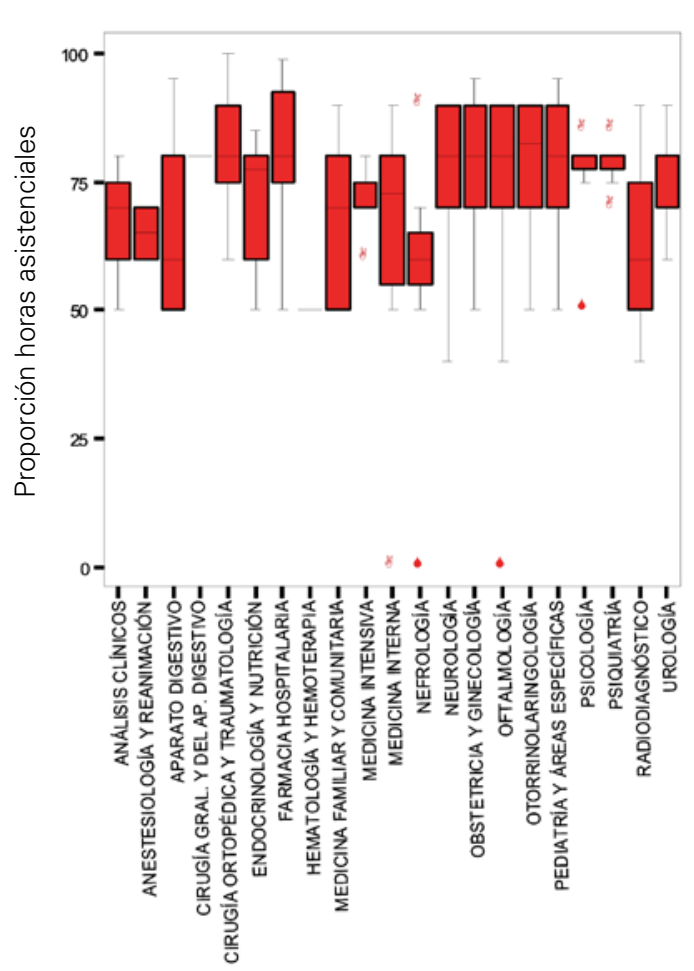

Especialidad MIR gunta ‘ $¿ E l$ hecho de hacer tantas guardias $-\mathrm{y}$ sus respectivas y preceptivas libranzas- perjudican su formación?', responden: en nada, 10,2\%; poco, 39,1\%; bastante, 29,7\%; mucho, 10,5\%, y no responden, 10,5\%. A la pregunta ¿¿En qué medida considera que las guardias son buenas para su formación como especialista?', responden: nada, $1,5 \%$; poco, $12,8 \%$; es lo adecuado, $36,5 \%$; bastante, $20,7 \%$; mucho, $21,4 \%$, y no responden, $7,1 \%$.

En definitiva, el $40 \%$ considera que un elevado número de guardias puede perjudicar a su formación (bastante + mucho). Por el contrario, más del $40 \%$ reconoce que las guardias son buenas para su formación (bastante + mucho). Esta contradicción probablemente refleja diversas opiniones sobre las guardias entre residentes de muy distintas especialidades.

\section{Del acceso a fuentes de información (incluyendo las fuentes electrónicas)}

¿En qué medida considera que las fuentes de infor- mación disponibles en su unidad docente resultan adecuadas y suficientes para su formación como especialista?': nada, 0,4\%; poco, 7,1\%; adecuado, $30,8 \%$; bastante, $43,6 \%$; mucho, $16,2 \%$, y sin respuesta, $1,9 \%$.

¿En qué medida considera que las fuentes de información disponibles en su unidad docente están fácil y rápidamente disponibles para la práctica asistencial?': poco, 9,4\%; adecuado, 30,8\%; bastante, 40,6\%; mucho, $17,4 \%$, y sin respuesta, $1,9 \%$.

¿En qué medida considera que el soporte de fuentes de información del hospital (fundamentalmente referido al soporte y servicio ofrecido por la biblioteca) resulta adecuado para su formación como especialista?': nada, $0,4 \%$; poco, $8,3 \%$; adecuado, $23,7 \%$; bastante, $42,1 \%$; mucho, $24,1 \%$, y sin respuesta, $1,5 \%$.

Las respuestas 'bastante' y 'mucho' suman en cada una de las preguntas un valor en torno al $60 \%$. Llama la atención que la respuesta 'poco' fue la opción para el 7-10\%. 


\section{De la supervisión de la actividad asistencial}

En la encuesta se plantearon unas modalidades o grados de supervisión de la actividad asistencial, que se describen en la tabla V. Se les solicitó que señalaran la frase con la que más identificaban el tipo o características de la supervisión experimentada en el año previo de formación. Concretamente se les dijo: 'Una visión global de la supervisión de la actividad asistencial en su unidad docente ha consistido principalmente en (seleccione la letra que más se aproxime a la afirmación, de entre las abajo señaladas). Se identifican con un nivel de supervisión A, 172 residentes (64,7\%); B, 16 (6\%); C, 48 (18\%); D, 2 (0,8\%); E, $12(4,5 \%)$, y sin respuesta, 16 (6\%). Ningún caso identificó la supervisión con la frase $\mathrm{F}$ (no existe prácticamente supervisión). Son las opciones A y C las más destacadas ( $65 \%$ y $18 \%$, respectivamente). Por tanto, el nivel de supervisión percibida, en general, parece adecuado. Nadie se pronunció por la opción F.

Respecto de la supervisión percibida por los residentes, observamos diferencia entre el colectivo de residentes de MFyC frente al resto de residentes de nuestro centro. Cuando hicimos al análisis del tipo de supervisión percibida, limitado a residentes de $\mathrm{MFyC}$, los resultados fueron como sigue: nivel de supervisión A, 40,7\%; B, 7,4\%; C, 22,2\%; D, 3,7\%, y E, $11,1 \%$. Tampoco ningún caso percibió la supervisión como prácticamente inexistente (nivel F). Y para el subgrupo del resto de residentes del HUPA, una vez excluidos los residentes de $\mathrm{MFyC}$, las cifras fueron: nivel A, 67,4\%; B, 5,9\%; C, 17,6\%; D, 0,4\%, y E, 3,8\%.

Parece pues que el nivel de supervisión percibida fue de un menor nivel en el caso del colectivo de MFyC. Así, al agrupar los niveles de supervisión en 'aceptable-alto' (A, B y C) o bajo (D, E y F), el análisis separado en los colectivos de MFyC frente al resto fue: entre $\mathrm{MFyC}$, el 70,4\% declararon percibir un nivel de supervisión aceptable-alto, mientras que entre el resto de residentes dicho nivel percibido fue del 90,8\% $(p<0,005)$.

\section{De la labor tutorial}

Sobre este aspecto se preguntó como sigue: 'En el año/curso objeto de esta evaluación, ¿su tutor de especialidad ha tenido alguna entrevista individual con usted centrada en su evolución como MIR y orientada a recibir feedback y a que plantee sus problemas e inquietudes?? Las respuestas fueron: no, 89 (33,5\%); sí, 166 (62,4\%), y sin respuesta, 11 (4,1\%). 'Si su respuesta ha sido no, ¿considera que debería haber tenido alguna entrevista en este sentido con su tutor?': respondieron sí 63 residentes, y no, 19.
Tabla V. Frases que describen el tipo de supervisión experimentado y con el que el residente identifica su experiencia en el año previo.

\begin{tabular}{ll}
\hline A $\quad$ El facultativo de plantilla supervisa mi actividad y asume las decisiones fundamentales \\
\hline B $\quad \begin{array}{l}\text { Es el MIR de curso o nivel superior de la especialidad quien más } \\
\text { a menudo supervisa mi actividad y asume las decisiones fundamentales }\end{array}$ \\
\hline C $\quad \begin{array}{l}\text { El facultativo de plantilla no ve habitualmente a los pacientes, } \\
\text { pero actúa de consultor tomando las decisiones, a requerimiento del residente }\end{array}$ \\
\hline D $\quad \begin{array}{l}\text { El residente de curso o nivel superior de la especialidad es quien, aunque no ve habitualmente } \\
\text { a los pacientes, actúa de consultor tomando las decisiones a requerimiento del residente }\end{array}$ \\
\hline E $\quad \begin{array}{l}\text { Existe supervisión ocasional, pero las decisiones fundamentales } \\
\text { son tomadas por mí o por otro residente de mi mismo nivel }\end{array}$ \\
\hline F $\quad$ No existe prácticamente supervisión \\
\hline
\end{tabular}

\section{De la acogida del residente de primer año}

Sobre este ítem se hicieron las preguntas siguientes únicamente a los residentes de primer año:

'De la acogida en el hospital, especialmente en lo que respecta a la información para poder desenvolverse como MIR, puede valorarla como...': excelente, $17,3 \%$; buena, $38,3 \%$; adecuada, $25,9 \%$; deficiente, $9,9 \%$, y muy deficiente, $2,5 \%$.

'De la acogida en la unidad docente, la información sobre todo lo necesario para iniciar su actividad como MIR (dependencias físicas, información técnica, bibliografía, protocolos, tipo de actividad a desarrollar) puede valorarla como...: excelente, $24,7 \%$; buena, 44,4\%; adecuada, 14,8\%; deficiente, 9,9\%, y sin respuesta, $6,2 \%$.

'La presentación a los diferentes facultativos y compañeros la califica como...': excelente, $48,1 \%$; buena, $32,1 \%$; adecuada, $13,6 \%$, y sin respuesta, $6,2 \%$.

'El grado de interés de los facultativos por la formación MIR, que usted percibió, podría calificarlo como...: excelente, $27,2 \%$; bueno, $48,1 \%$; adecuado, $17,3 \%$; deficiente, $1,2 \%$, y sin respuesta, $6,2 \%$.

Creemos poder afirmar que, en general, la acogida del centro y en las unidades es bien percibida, aunque para un 10-12\% es manifiestamente mejorable.

\section{Análisis de los comentarios abiertos}

Para el análisis de este apartado se ha procurado agrupar lo mejor posible las ideas expresadas por los residentes en comentario libre. Se han analizado 76 comentarios abiertos. En la tabla VI, que resume las ideas expresadas, se han eliminado comentarios 
muy específicos para algún colectivo de residentes y se han mantenido los que parecen afectar a una elevada proporción de éstos. Aun así, es evidente que algunos de los comentarios incluidos afectan a pocos residentes, pero pueden ser ilustrativos de una forma de ver su realidad. Por último, esta tabla no deja de ser un análisis cualitativo personal y podría haberse construido de forma diferente.

\section{Discusión}

Hemos obtenido una puntuación razonablemente buena para todas las unidades docentes. Son excepcionales los residentes que califican la formación en su unidad docente como deficiente. Casi en el $50 \%$ de las unidades docentes tienen lugar sesiones clínicas más de una vez a la semana e incluso diarias, sesiones a las que pueden asistir mayoritariamente y en las que participan los residentes de forma masiva. Respecto del número de sesiones clínicas que presentan, debemos hacer una reflexión ante la pregunta que se les hizo, pues no parece existir un consenso unánime de lo que se entiende por sesión clínica, o bien este concepto es muy diferente en cada servicio, lo que conlleva una gran desviación estándar en la media de sesiones que dicen presentar cada uno de ellos $(11,2 \pm 41$ o bien 4,7 $\pm 5,5$ cuando se excluyen valores extremos). Mayoritariamente se sienten respaldados cuando tienen que presentar sesiones clínicas o participaciones en congresos. No obstante, un $8-10 \%$ sienten un escaso o nulo apoyo para tales tareas.

El 34\% de los residentes dice no haber participado en colaboraciones a congresos en los años evaluados. La media anual es de 2,2 comunicaciones, también con amplia desviación estándar $( \pm 3,4)$, cifras que se han mantenido estables a lo largo de los tres años del estudio. La amplitud de la dispersión de la cifra media refleja diferencias apreciables por años de residencia (lógicamente contribuyen a congresos más a medida que avanzan en su proceso formativo) y por unidad docente.

La proporción de tiempo dedicado a la asistencia y el promedio de guardias realizadas $(5 \pm 1) \mathrm{pa}-$ rece dentro de lo razonable, sin grandes diferencias entre unidades docentes. La vivencia de las guardias como positivas o negativas para la formación es dispar y probablemente refleja diferencias notables entre especialidades.

La accesibilidad a las diferentes fuentes de información en general resulta buena o muy buena, si bien un $7-9 \%$ de residentes consultados declaran escasa accesibilidad a ellas, lo que merecería algún tipo de reflexión sobre el aprendizaje para mejorar su utilización.

Respecto del importante punto de la supervisión de su actividad asistencial, intrínseco al sistema de formación especializada en nuestro país [3,9], los residentes afirman sentir un grado de supervisión que podríamos considerar elevado o adecuado. No obstante, existen diferencias notables y estadísticamente significativas cuando la cuestión sobre la supervisión percibida por el residente se dirige sólo a residentes de MFyC frente al resto. Ello probablemente se debe a que una parte muy importante de la actividad asistencial de los residentes de esta especialidad tiene lugar en el ámbito de la urgencia, lugar donde probablemente la supervisión puede ser más débil.

Un tema también de particular importancia es el de las entrevistas personalizadas tutor-residente, que deben tener lugar a lo largo del año. Según el Real Decreto 183/2008, deben producirse una vez al trimestre, ser sistemáticas y dejar constancia de que han tenido lugar. En nuestro estudio únicamente se preguntó si habían tenido alguna entrevista individual con su tutor. El 33\% dijeron que no. Esto debe corregirse inmediatamente y confiamos que ante esta pregunta la respuesta negativa sea la excepción a partir del curso 2009-2010. Para ello, la CLD de nuestro centro aprobó, a primeros de 2010, un formato para orientar las entrevistas semiestructuradas tutor-residente que se presentó en la VII Jornada del Pleno de la Red de Comisiones de Docencia y Asesoras de la Comunidad de Madrid (REDFESMA) [10]; su implantación en la práctica acaba de comenzar en nuestro centro.

La acogida en las unidades docentes y del hospital en general se percibe como adecuada o buena, aunque un $8-12 \%$ piensan que no ha sido así, lo que debería hacer reflexionar también sobre nuestras posibilidades de mejora en el procedimiento de acogida. Probablemente deberemos empeñarnos en mejorar lo referente a la información relacionada con importantes aspectos de la tarea y el entorno en que se inician en su vida profesional, suponiendo que ello puede ser causa de esta escasa proporción de residentes que declaran insatisfacción en estas preguntas.

Respecto de la tabla de comentarios libres, pensamos que de su lectura cada cual puede extraer conclusiones interesantes. Las guardias y las urgencias en general siguen siendo percibidas como un problema y como un lugar donde quedan aspectos de mejora importantes. Resaltar también la necesidad de mejorar la capacitación del residente para el acceso a fuentes de información, la incorporación 
Tabla VI. Análisis-resumen de los comentarios abiertos expresados libremente por los residentes

\section{Guardias}

Disminuir guardias fue el comentario más frecuente: 21 residentes lo expresan, en 12 de ellos como único comentario

Otros comentarios aislados sobre guardias:

Más guardias de área médica y menos de área quirúrgica

Sancionar la falta de asistencia a guardias

Librar las guardias

Disminuir rotaciones de guardia en centro extrahospitalario

Urgencias

Aproximadamente 17 comentarios respecto del funcionamiento y organización de la urgencia:

Deficiente formación en urgencias

Varios comentarios como: mayor protocolización, organización y personal en la urgencia; mejor organización y distribución de los residentes en la urgencia (dos comentarios)

Mejor organización de las guardias; más coordinación de guardias entre el servicio de urgencias y planta

Varios comentarios como: más adjuntos en la urgencia, mayor presencia de adjuntos en la urgencia, más supervisión y también 'nula supervisión en urgencias', 'en cirugía, el MIR-I, por la noche, está solo', 'se firman altas sin supervisión'

Necesidad de rotación en la urgencia junto con un residente mayor. No superar dos meses seguidos de rotación por la urgencia

Emplear más tiempo en los pacientes encamados de la urgencia (dos comentarios)

\section{Docencia propiamente dicha}

Aproximadamente entre 18 y 20 comentarios referidos a 'docencia' y expresados de formas variadas:

Mayor tiempo dedicado a la docencia; solicitar más docencia a la unidad docente

Necesidad de un programa de contenidos (dos residentes así lo expresan)

Más docencia exclusiva a psicólogos internos residentes (dos residentes lo expresan)

Mejorar la docencia en el quirófano

Sesiones acerca de los casos más interesantes, mayor número de sesiones clínicas, sesiones clínicas para residentes, organizar un mínimo de sesiones clínicas al inicio del curso

Más talleres con maniquíes y simulación

Compartir docencia con otros servicios hospitalarios

Aumentar el número de clases semanales, regularizar el horario de clases; mayor flexibilidad en el horario de los cursos

\section{Investigación y congresos}

Aproximadamente 10 comentarios como:

Mayor colaboración de adjuntos para publicaciones y sesiones

Fomentar la investigación (varios comentarios), más trabajos de investigación con soporte técnico y económico (un comentario)
Apoyar y fomentar publicaciones (dos comentarios)

Mayor asistencia a congresos, poder asistir al congreso anual, ayuda económica para congresos, 'no asistimos a congresos'

\section{Más actividades formativas o cursos de formación}

Uno de los comentarios frecuentes, en al menos ocho casos

\section{Sobre fuentes de información}

Cinco comentarios siguientes:

Cursos de búsqueda de documentos científicos por Internet (dos comentarios)

Acceso libre desde el centro al programa SPSS

Más ordenadores en la biblioteca

Actualización bibliográfica

\section{Evaluación del proceso formativo en general}

Mayor evaluación de la calidad de formación

Mayor información sobre decisiones tomadas en la evaluación (dos comentarios)

Tomar medidas en base a los resultados de estas encuestas

Reevaluar las rotaciones

Supervisión y tutoría en general

Varios comentarios como:

Mayor supervisión por parte del tutor (cuatro comentarios)

Mayor supervisión de facultativos en el paritorio

Aumentar el número de tutores

Mejorar la función de sus tutores

Mejor organización por parte del tutor

Trabajar con distintos tutores en el centro de salud

Más colaboración y supervisión de los adjuntos

\section{Sobre actividad quirúrgica}

Mayor participación en las actividades de cirugía

Operar más

Más cirugía con residentes

Participar en las intervenciones quirúrgicas también por la tarde

\section{Miscelánea}

Tener entrevistas interpersonales adjunto-residente

Tener sesión de residentes con tutor y otro adjunto

Más participación del residente en las consultas (dos comentarios), dedicar más tiempo a la planta y las consultas

Promover rotaciones en el extranjero

Facilitar rotaciones externas 
de residentes a la investigación y la supervisión de residentes en general, así como incluir entrevistas personalizadas y mejorar el procedimiento general de evaluación.

Nuestro estudio es pionero en España, si bien recientemente se han llevado a cabo algunos trabajos sobre este aspecto [11,12], y aunque algunas de sus conclusiones son comunes a las obtenidas por nosotros, este trabajo aporta una perspectiva de tres años de seguimiento.

Este estudio debe servir para implantar acciones orientadas a mejorar las deficiencias observadas en aras de una mejora continua de la calidad de la formación de residentes. Consideramos que este estudio, además, constituirá un punto de referencia para conocer la evolución año a año del proceso de enseñanza/aprendizaje en nuestro centro. Sin este tipo de estudios resulta difícil conocer los progresos o deficiencias que, desde el punto de vista de quien aprende, tienen lugar en un determinado ámbito de la formación de especialistas en ciencias de la salud.

En conclusión, la reflexión sobre la formación de los residentes es una necesidad dentro de una formación de calidad; sólo desde ella se pueden planificar áreas de mejora. La opinión de los que se forman es un aspecto fundamental del proceso de reflexión. La pregunta directa a quien se forma, su registro y su evaluación son fundamentales. La evaluación crítica de lo que hacemos para formar a los residentes resulta esencial para una mejora continua del proceso.
Bibliografía

1. Rodríguez-Neira T. La evaluación en el aula. Madrid: Nobel; 2000.

2. Bain K. Lo que hacen los mejores profesores universitarios. Valencia: PUV; 2004.

3. Real Decreto $183 / 2008$, de 8 de febrero, por el que se determinan y clasifican las especialidades en ciencias de la salud y se desarrollan determinados aspectos del sistema de formación sanitaria especializada. BOE, n. ${ }^{\circ}$ 45, de 21 febrero de 2008.

4. Pardell H. El médico del futuro. Barcelona: Fundación Educación Médica; 2009.

5. Ruiz-Moral R Aspectos generales sobre la evaluación. In Ruiz-Moral R, ed. Educación médica. Manual práctico para clínicos. Madrid: Panamericana; 2009. p. 223-7.

6. Evans RG, Edwards A, Evans S, Elwyn B, Elwyn G. Assessing the practicing physician using patient surveys: a systematic review of instruments and feedback methods. Fam Pract 2007; 24: 117-27.

7. Overeem K, Faber MJ, Arah OA, Elwyn G, Lombarts KM, Wollersheim HC, et al. Doctor performance assessment in daily practice: does it help doctors or not? A systematic review. Med Educ 2007; 41: 1039-49.

8. Orden de 22 de junio de 1995 por la que se regulan las comisiones de docencia y los sistemas de evaluación de la formación de médicos y de farmacéuticos especialistas. BOE, n. ${ }^{\circ} 155$, de 30 de junio de 1995

9. Ley 44/2003, de 21 de noviembre, de ordenación de las profesiones sanitarias. BOE, n. ${ }^{\circ} 280$, de 22 de noviembre de 2003.

10. Gómez-Carrasco JA. La entrevista estructurada tutor-residente en la evaluación formativa. VII Jornada del Pleno de la Red de Comisiones de Docencia y Asesoras de la Comunidad de Madrid. Madrid, junio de 2010.

11. Llompart-Alabern I, Escobar-Oblitas D, Cremades-Maestre AI Manuel Rimbau-Muñoz E, Vidal-Puigserver J. Resultados de la encuesta de satisfacción percibida por los residentes sobre la docencia recibida durante el año 2009 en el Hospital Universitario Son Dureta de Palma de Mallorca. Actas del VIII Encuentro de Tutores y Jefes de Estudio de la Formación Especializada. Menorca, septiembre de 2009.

12. Hernández A, Losada A, Brotons A, Buades J. Visión de la residencia por los MIR: problemas y soluciones. Actas del VIII Encuentro de Tutores y Jefes de Estudio de la Formación Especializada. Menorca, septiembre de 2009. 